\title{
Context-Aware Recommendations through Activity Recognition
}

\author{
Toon De Pessemier, Simon Dooms, Kris Vanhecke, Bart Matté, Ewout Meyns, Luc Martens \\ WiCa research group, Dept. of Information Technology, iMinds-Ghent University \\ Gaston Crommenlaan 8 box 201, B-9050 Ghent, Belgium \\ \{toon.depessemier, simon.dooms, kris.vanhecke, luc.martens\}@intec.ugent.be
}

Keywords: Context-Aware, Recommender System, Activity Recognition, Mobile

\begin{abstract}
The mobile Internet introduces new opportunities to gain insight in the current user's environment, behavior, and activity. This additional contextual information can be used as an extra information source to improve traditional recommendation algorithms. This article describes a framework to detect the current context and activity of the user by analyzing data retrieved from different sensors available on mobile devices. The framework can easily be extended to detect custom activities and is built in a generic way to ensure easy integration with other applications. On top of this framework, a recommender system is built to provide a personalized content offer, consisting of relevant information such as points-of-interest, train schedules, and touristic info, based on the user's current context. Users who tested the application confirmed the usability and liked to use it. The recommendations are assessed as effective and help them to discover new places and interesting information.
\end{abstract}

\section{INTRODUCTION}

Contextual information is used in many application domains to offer users a service that is adapted to their location, needs, and expectations. Also in recommender systems, the user context has gained an increased interest from researchers (Adomavicius et al., 2005). For instance, various tourist guide applications use the location of the user to personalize and adapt their content offer to the current user needs. An interesting example is a mobile recommender system proving personal recommendation for Points of Interest (POI) based on the user ratings (Kenteris et al., 2010). User ratings can be weighted higher to differentiate between users that rate POIs using the mobile tourist guide application in direct proximity of the POI and others using the Internet away from the POI. Still via mobile devices such as smartphones, more contextual information can be retrieved than currently exploited by traditional recommendation algorithms. Users are carrying their mobile device on them, resulting in additional information such as their location, speed, environment, etc. This additional information can revolutionize the role of recommender systems from topic oriented information seeking and decision making tools to information discovery and entertaining companions (Ricci, 2010).

To ease the development of mobile context-aware applications, frameworks have been introduced to provide an abstraction for sensors and actuators. Such a framework assists application developers in gathering data from various sensors, represent application context, and reason efficiently about the context, without the need to write complex code (Biegel and Cahill, 2004). However, most of these frameworks provide only low-level sensor data and do not interpret the data over a longer period of time to deduce high-level context information such as the user's activity.

Various attempts have been made to recognizing user activity from accelerometer data. Wearable sensors have been used to measure acceleration and angular velocity data in order to recognize and classify sitting, standing, and walking behaviors (Lee and Mase, 2002). An experiment with five biaxial accelerometers worn simultaneously on different parts of the body, showed that it is possible to recognize a variety of different activities like walking, sitting, standing, but also watching TV, running, bicycling, eating, reading etc. (Bao and Intille, 2004). Moreover, the recognition performance drops only slightly if data of only two biaxial accelerometers is available - thigh and wrist.

Also through a single triaxial accelerometer worn near the pelvic region, user activities can be recognized with fairly high accuracy. Nevertheless, ex- 
periments showed that activities that are limited to the movement of just hands or mouth (e.g. brushing teeth) are comparatively harder to recognize using a single accelerometer (Ravi et al., 2005). Although most mobile devices contain only a single triaxial accelerometer, these results indicate the ability to detect user activities through this built-in accelerometer.

In this research, we present a framework to recognize the user's context and activity based on sensor data originating from the user's mobile phone in a daily user environment. The developed framework (Section 2) first detects basic contexts and activities such as walking and cycling by analyzing the acceleration of the mobile device. By analyzing these basic activities over a longer period of time, recognizing more complex contexts such as "walking to a station while it is rainy" is possible. This contextual information is used by the recommender system (Section 3 ) in order to achieve the main goal of this research: providing personalized information and suggestions that are adapted to the current context and activity of the user. The accuracy and usefulness of the recommendations is assessed via a user study (Section 4).

\section{RECOGNITION FRAMEWORK}

Because of its rapid growth in popularity and widespread use, we opted for Google Android as implementation platform of our framework. Nowadays, almost every Google Android device has several builtin sensors, such as an accelerometer and GPS. But sensor data is also available in many other operating systems for mobile devices.

The context-recognition framework consists of three successive phases: 1. Monitoring the (sensor) data, i.e. logging the raw data from the accelerometer, GPS, battery, proximity sensor, cell ID, etc. 2 . Processing the sensor data and recognizing basic activities. 3. Analyzing the successive basic activities and recognizing the overall context.

\subsection{Monitoring}

This phase involves the gathering of all possible raw data from the device. GPS data provides location updates. If no GPS data is available (e.g., in indoor environments), the cell-ID can give an indication of the location through the ID of the cellular tower that is currently providing reception to the device. Further, the battery status (e.g., charging) of the device as well as the battery level can be retrieved. The accelerometer of most Android devices is capable of capturing the device's acceleration on three axes every $20 \mathrm{~ms}$.
This accelerometer data is used to recognize the activity of the user. The proximity sensor is used by the Android operating system to detect if an object is in the vicinity of the device. Its main purpose is to detect if the user is holding the device next to the ear for making a phone call. In that case, the screen can be switched off to save power. In this research, the proximity sensor is used to detect where the user carries the device. If the proximity sensor detects no object in the vicinity of the device, then the device is not in the pocket of the user, and recognizing basic activities based on accelerometer data is not reliable. The framework can easily be extended with additional sensor data in order to add additional contextual information.

\subsection{Processing}

In this phase, each type of data obtained in the monitoring phase, is converted into basic contextual information by a processing unit. For some sensor data, such as data from the proximity sensor, this conversion is straightforward. Other sensor data, such as data from the accelerometer, requires a more intelligent processing to obtain contextual information. If additional sensor data becomes available, the framework can be extended with a new processing unit to extract valuable information from it.

\subsubsection{Points-of-interest}

Matching the current location of the user to the location of Points-Of-Interest (POI) enables the framework to identify the nearest POI or the POI within a specified range. The location of the user is retrieved via GPS data or (if GPS is not available, or switched off) estimated by the current cell-ID. Two different services are used to retrieve data about the POI in the current neighborhood of the user. The location of the Belgian railway stations is retrieved via the iRail API (Tiete et al., 2012), a service that provides information about train stations, schedules, and delays in Belgium. Via the Foursquare API (Foursquare, 2012), the framework retrieves data about various other types of POI such as restaurants, bars, shops, etc.

\subsubsection{Urbanization}

The POI that are retrieved by the Foursquare API are used to estimate the urbanization of the current location of the user. The more POI in the neighborhood of the device, the higher the urbanization level of the neighborhood. 


\subsubsection{Weather}

To find out the weather conditions, the location of the user is first converted into an address via the Google Geocoding API (Google, 2012a). Subsequently, the ZIP code of the address is used to retrieve weather information from the Google Weather API. This information is refreshed after a change in location or if more than 2 hours have elapsed. To retrieve data about the current weather and urbanization level, GPS data is not strictly required since an estimation of the location of the device by the cell-ID is sufficiently accurate.

\subsubsection{Movement}

Based on location updates of the GPS data (or cellID info) and the coupled timestamps, the framework calculates the current speed and future position of the user. Together with the information about the POI, the framework can detect if the user is approaching a POI.

\subsubsection{Company}

In the application, users can add other users as friends and specify their relationship with these friends, e.g., husband, child, buddy etc. Besides, users can opt to share their location data in order to enable the framework to detect whether different users are in another's company or whether some of their friends are in the neighborhood.

\subsubsection{Available Time}

By checking the user's appointments in the calendar application of the phone, the framework can estimate the availability of the user. Appointments in the near future can influence the behavior of the user. E.g., if the user has an appointment within one hour, (s)he might chose a nearby restaurant to have lunch.

\subsubsection{Battery}

Information about the status of the battery can be used to deduce contextual information of the user, e.g., charging the battery indicates a fixed position of the user. (Many users charge their phone while they are at home.) Data about the battery level can be used to decide to switch off the framework to extend the battery lifetime.

\subsubsection{Physical activity}

Recognizing physical activities based on patterns in the data originating from the accelerometer is the most complicated processing task of the framework. The framework tries to distinguish four basic activities: standing still, walking, running, and cycling. These different activities induce different accelerations along the three dimensions (X-axis, Y-axis, and $\mathrm{Z}$-axis); and these patterns in the accelerometer data are used to distinguish the basic activities. An important requirement is that users have to carry the mobile device in their pocket, so that the movement of the user's leg can be registered by the device.

Learning to recognize patterns in the accelerometer data is done by training the framework with samples of real physical activities. To obtain this training data, accelerometer data from 11 different users (between 16 and 50 years old) performing the four activities was collected. Every user was asked to perform one of the basic activities during a 5-seconds time frame while a mobile device recorded the accelerometer data. This was repeated for all four basis activities, thereby yielding 44 training samples. This training data clearly showed different patterns for the four activities: e.g., standing still induces the least activity on the accelerometer, cycling produces a data pattern with a periodic variation in time, and running shows more energy than walking.

This training data was used for determining the five discriminating features based on which the four basic activities are distinguished:

1. The average resultant acceleration, i.e. the average of the square root of the sum of the values of each axis squared $\sqrt{x_{i}^{2}+y_{i}^{2}+z_{i}^{2}}$.

2. The difference between maximum and minimum acceleration (for each axis).

3. The average deviation to the mean (for each axis), i.e. the average of the absolute difference between a measured sample of the acceleration and the mean acceleration.

4. The sum of the squared deviations to the mean value (for each axis), i.e. the sum of the squared differences between a measured value of the acceleration and the mean acceleration.

5. The deviation of the acceleration (for each axis), i.e. the average of the absolute difference between a measured sample of the acceleration and the sample measured after three time units (so after 60ms).

The first three of these discriminating features were also identified in related work with respect to activity recognition on mobile devices (Kwapisz et al., 2011). Discriminating feature (4) and (5) help to distinguish the basic activities based on typical characteristics 
such as the required energy for the activity and the variation of the acceleration in time.

Based on these discriminating features, newlyacquired accelerometer data can be classified into one of the basic activities. This classification task is performed by using Support Vector Machines (SVM) with an RBF-kernel. Using cross validation thereby considering the data from 1 user as test data and the data from the other users to train the model, each of the 44 logged activities could be classified correctly by the SVM model.

\subsubsection{Proximity}

As explained in Section 2.1, the data of the proximity sensor can indicate that the device is not in the pocket of the user. Since the recognition of physical activities requires the user to carry the device in his/her pocket, this proximity data can indicate if the activity recognition is reliable.

\subsection{Analyzing}

Based on the basic activities that are recognized by processing the accelerometer data and the additional contextual information gathered in the processing phase, the framework can recognize more complex user behavior. The underlying idea of the analyzing phase is that complex user behavior consists of different basic contexts which have some relation with each other. E.g., "The user is walking home while it's rainy" consists of "The user is walking", "The user is approaching his/her house" and "it's rainy". The common conditional relationship between these basic contexts is the timing; they have to occur at the same time.

So to recognize complex user behavior, these complex activities are first decomposed into different basic contexts that have a conditional relationship to each other. A basic context can be: the current weather, the current time and day, the battery status and level, being located in an urbanized area, being located in the neighborhood of a specific POI, approaching a specific POI, being in the company of another user, traveling with a specific speed (range), the distance traveled in a specific time interval, or a physical activity such as standing still, walking, running, or cycling. For each potential complex activity, the framework checks if the first basic context matches the data that is gathered in the processing phase. If this is the case, the framework checks the conditional relationship of this basic context to the second basic context. The conditional relationship can indicate that the second basic context has to occur in parallel with the first basic context or within a specified time frame (e.g., within the next 60 minutes after the first context was detected). So upon detecting the first context, until the conditional time frame has elapsed, the framework monitors the sensor data and tests if the processed data matches the pattern of the second basic context. This procedure of matching the processed sensor data to the basic contexts and testing the conditions, is repeated for all basic contexts and conditions of the complex activity.

As soon as one of the basic contexts of the complex activity cannot be matched to the processed sensor data or one of the conditions between the basic activities is not met, the complex activity cannot be recognized. Only if all basic contexts are recognized and all conditions are met, the complex activity is flagged as recognized.

An example of a complex activity is "taking the train" which is composed of the following subsequent basic contexts: 1) The user is approaching a train station. 2) The user is in the neighborhood of a train station. 3) GPS connection is lost. (Although GPS data is available inside a car, GPS data is not available inside the train). 4) The user is traveling with a minimum speed. (In this case, location updated are based on cell-ID, because GPS info is not available.) 5) In parallel with 4), the user is traveling in the direction of another (nearby) train station. As soon as these basic contexts and conditions are recognized, the framework believes that user is traveling by train. This complex activity does not include the act of arriving at the train station of the destination. If the destination would be included in the complex activity, then the activity could only be recognized after the train journey. Nevertheless, for many applications such as personalized information and recommendations, the recognition has to be performed as soon as possible during the user activity. In the current implementation, a set of complex activities is defined, but depending on the use case, the framework can also be extended with new complex activities by composing existing or new basic contexts and conditions.

\section{CONTEXT-AWARE RECOMMENDATIONS}

Based on the contextual information that is provided by the context-recognition framework, we developed a context-aware recommender system that offers personalized information according to the preferences and current context of the user.

To enable the use of community knowledge (i.e. data regarding user behavior, feedback, and contextual information from all users of the system) in the 


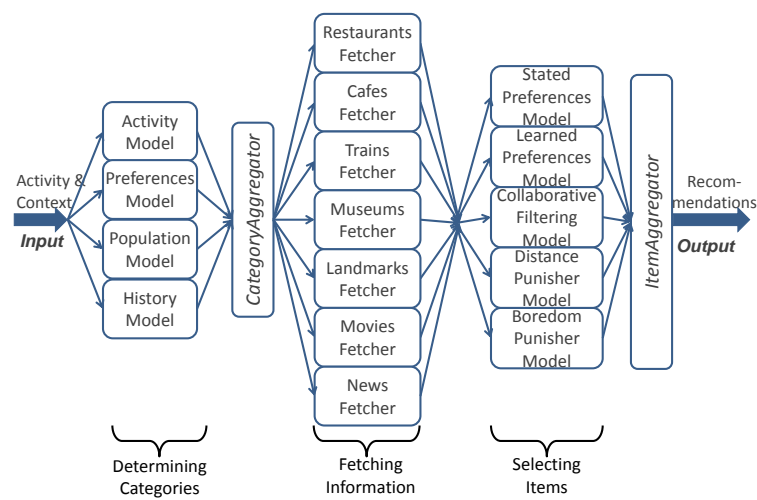

Figure 1: Schematic overview of the recommendation process, which consists of three successive phases: determining the categories, fetching the information, and selecting the items.

recommendation process, personalized recommendations are calculated on a centralized server. Based on this community knowledge, Collaborative Filtering $(\mathrm{CF})$ techniques can be used to assist in the recommendation process (Breese et al., 1998). The mobile client can send the server a request for recommendations combined with the current context that is retrieved from the context recognition framework. Based on the stored preferences of the user and the contextual information, the server calculates the most appropriate context-aware recommendations tailored to the user's (current) needs.

As shown in Figure 1, the recommendation process consists of three successive phases:

1. Determining the categories of information that are most suitable according to the current context of the user.

2. Fetching the information of the items of these selected categories.

3. Selecting the most suitable items from the retrieved information according to the context and preferences of the users.

After determining the categories and selecting the items, an aggregator combines the partial results.

\subsection{Determining the categories}

In the first phase, the recommender system receives the current context of the user as input, and predicts the information categories that match this context. One obvious example: if the user is approaching a train station, information regarding the train schedule might be interesting for the user. To determine the suitability of an information category, four information models work together: the activity model, pref- erences model, popularity model, and history model. Each of these models assigns a probability score to each information category. This score estimates the conditional probability that the user is interested in information of the specific category, given the current context of the user. The information categories that are used are: Food (restaurants, bakeries, etc.), Movies (schedules, descriptions, etc.), Trains (schedules, delays, etc.), Monuments (info about churches, statues, etc.), and News (newspaper articles, RSS feeds, etc.); but the system can easily be extended with other categories.

\subsubsection{Activity model}

The activity model is a knowledge-based system, consisting of a set of general rules that apply to all users. These rules connect a context to an information category that may be interesting for the user in that context. E.g., the context "being in a new city" and "sunny weather" is linked to the information category "Monuments", since users might be interested to do some sightseeing if the weather is good. The context "Evening" is linked to the information category "Food", since information about restaurants for having dinner might be interesting. These rules are stored as triplets (context, category, score), in which the score estimates the probability that users are generally interested in a category, given the specified context.

These general trends of the activity model also offer a solution to the cold start problem, the initial situation in which no information about the preferences of the user is available. If no personal preferences are known, the user receives recommendations based on the knowledge of these general trends.

\subsubsection{Preferences model}

The activity model defines rules for the whole community; but via the preferences model, rules can be specified for each individual user. This way, user preferences for a specific information category, given a specific context, can be specified. E.g., user "Alice" always wants to receive items of the category "News", if she is traveling by train in the morning. These personal rules are stored as 4-tuples (user, context, category, score), in which the score indicates how important this rule is for the user. An initial explicit questionnaire can be used as input to compose these rules.

\subsubsection{Popularity model}

This model keeps track of the historical behavior of users and learns in which information categories users 


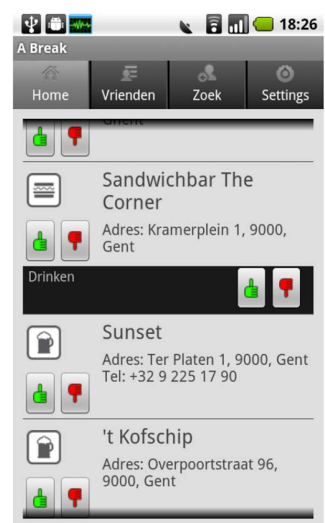

(a)

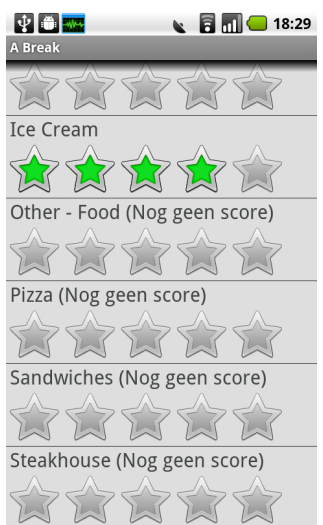

(b)
Figure 2: Screenshots of the mobile application, showing the list of recommended information items (a) and the possibility to provide explicit feedback (b).

are interested, given the context. This learning process is based on the feedback that users can provide for information categories. Figure 2 shows two screenshots of the user interface of the mobile application and illustrates the possibility to provide feedback. The popularity model collects feedback information from all users to discover general relations between a context and an information category. The result of this model is a set of triplets (context, category, score) in which the score estimates the probability that users are generally interested in a category, given the specified context. The more users and the more often these users have provided positive/negative feedback on a content item of a specific category in a specific context, the higher/lower the score.

\subsubsection{History model}

In contrast to the popularity model, which learns category preferences for different contexts on the community level, the history model learns category preferences for each context on a user level. The model aggregates the historical behavior of each user into a profile to learn the user's personal practices. E.g., user Alice may be interested in the train schedule as soon as she leaves her home in the morning. So, the history model calculates for every user, context, and category, a score which estimates the probability that a specific user is interested in a category, given the context. The more often the user has provided positive/negative feedback on a content item of a specific category in a specific context, the higher/lower the score. These personal habits are stored as 4-tuples (user, context, category, score).

\subsubsection{Aggregating the category scores}

Each of the models generates its own score which estimates the probability that the user is interested in a specific category, given the specified context. To obtain a single probability value for each category, the individual scores are normalized and aggregated using a weighted average. In the current implementation, the weights are fixed and set to prioritize the models that reason based on data of the individual users, i.e. the preferences and history model. However, one can argue that varying weights might be more efficient: assigning more importance to the models that are based on community knowledge if a limited amount of personal preferences are available; and raising the importance of the models that use individual data if more knowledge about the user's individual preferences becomes available.

The resulting scores determine the importance of each information category for the user. Therefore, the user receives a proportional number of items of a specific information category as recommendations. Items of an information category with a high score are more common in the recommendation list, whereas items of an information category with a low score are rare or even not present in this list.

\subsection{Fetching the information}

As soon as the most suitable categories are determined, given the preferences and context of the user, information items belonging to these categories can be fetched. Because this information has to be upto-date (e.g., for the train schedule or newspaper articles) and because this information is dependent on the context of the user (e.g., the neighboring POI are determined based on the current location of the user), the information items are retrieved at the moment of requesting the recommendations.

Various services are used to fetch information items of the different information categories. Information regarding locations or POI (e.g., information about monuments, restaurants, shops, bars, trains, etc.), is selected based on the current location of the user. Potentially interesting data about nearby train stations, train schedules, and delays is retrieved via iRail (Tiete et al., 2012). Information about POI in the current neighborhood of the user is retrieved via the Foursquare API (Foursquare, 2012) and the Google Places API (Google, 2012b). WikiLocation is the service that is used for additional information about monuments and landmarks that might be interesting for the user (Dodson, 2012). Information about (cultural) events is available through the service 
of CultuurNet Vlaanderen (CultuurNet-Vlaanderen, 2012). CultuurNet gathers all information about cultural activities, movies, and events in Flanders (i.e. the Northern part of Belgium). This service is used to retrieve e.g., information about movie theaters and the scheduled movies. Various comparable services that offer news feeds exist. Because of its structured metadata, the RSS feed of HLN (HLN, 2012) is used to obtain the latest news articles of different categories such as sports, business, local news, international news, etc.

\subsection{Selecting items}

The last phase of the recommendation process is to select the most appropriate items from the fetched information of the relevant categories. To accomplish this task, five models for selecting items cooperate: the collaborative filtering model, stated preferences model, learned preferences model, distance punisher model, and boredom punisher model. Each of these models assigns a score for the usefulness to each item, thereby indicating how interesting or important the item is for the user. Some of these models consider the preferences of the user, whereas others are merely based on the current context of the user.

\subsubsection{Stated preferences model}

Through explicit feedback for an item or an attribute of the item, users can state their preference for a particular item (e.g., the user's favorite restaurant) or for a set of items characterized by the attribute they have in common (e.g., all Italian restaurants). In the user's profile, explicit feedback for an item propagates to the attributes and the category of the item. E.g., a positive evaluation of a news article about soccer induces a positive assessment for the attributes "Sports" and "Soccer" as well as for the category "News". As shown in Figure 2, users can specify these preferences via a star-rating mechanism in the user interface, thereby creating a personal profile consisting of triplets (user, item or attribute, score). Based on this explicit profile, the stated preferences model assigns a score to each candidate item by considering the user's rating for the item and/or the attributes describing the item. The context is not considered in the stated preferences model because of the large number of combinations of context and attribute. Specifying preferences for all these different context-attribute combinations can put a heavy burden on the user.

\subsubsection{Learned preferences model}

Whereas the stated preferences model is based on explicit preferences for items and attributes of items, the learned preferences model extracts these preferences from implicit data and learns the user behavior. By saving the implicit preferences as 4-tuples (user, context, item or attribute, score), this model can also take into account the context of the user. This way, the recommender can learn for example that the user likes fast-food for lunch, a hot soup on a cold winter day, or a soda after running. Also in this model, feedback for an item propagates to the attributes and the category of the item.

Implicit feedback is gathered by tracking the user's location. If the user is approaching a POI, such as a restaurant or a pub, the framework will monitor the time that the user is staying at that POI. Together with the number of visits to the POI, this data provides some insights into the user's preference for the POI. The more a user visits a POI, and the longer (s)he stays there, the better the implicit feedback for that item. In the current implementation, the implicit feedback is a linear function of the time of a visit and the number of visits, in which the coefficients are determined by the information category of the item. For items of the category "News", implicit feedback is based on the view-time of an article.

\subsubsection{Collaborative filtering model}

This model predicts a score for each item by using a standard user-based collaborative filtering algorithm, thereby yielding triplets (user, item or attribute, score). Collaborative filtering is a technique to estimate the preferences of a user for not-evaluated items, by using the preferences of many similar user for these items. These similar users are defined as users with similar preferences on a set of previouslyevaluated items and are identified by using a similarity metric (Breese et al., 1998). Here, the Pearson correlation metric is used for calculating similarities. Using the preferences of the community, the collaborative filtering model assigns the highest scores to the items that best match the preferences of the user, but neglects thereby the contextual information.

\subsubsection{Distance punisher model}

Since the recommender system has to suggest location-based items, such as restaurants, shops, train info, or the cinema schedule, the location of these items with respect to the current location of the user is especially important. The rational behind the distance punisher model is the users' preference for nearby 
items. E.g., if the user is traveling on foot, faraway places are not attainable and recommendations for these places are undesirable. Therefore, this model favors items in the direct neighborhood of the user at the expense of more distant places.

The distance that the user is willing to cover in order to reach a POI depends on the travel mode of the user. By bicycle, the user can move faster than on foot; and by car or train, even distant places can be reached. So the physical activity of the user is important contextual information that is used in the distance punisher model. Also the weather is a contextual aspect that influences the distance that users are willing to cover. Traveling on foot or by bicycle in combination with snow or rain will strengthen the users' preference for nearby places; whereas in sunny weather conditions, users might like to walk to their destination.

A measure of the accessibility of a place can be obtained by using distance decay curves for the different travel modes. For multiple travel modes and different purposes, the distance decay function fits a negative exponential curve, as demonstrated by research focusing on the detailed relationship between actual travel behavior and the mean distance to various services (Iacono et al., 2008). However these proposed distance decay functions cannot be adopted in this research (without changes), since the weather is not included as contextual parameter.

So in this research, the usefulness of an item was estimated by a negative exponential function of the distance, $d$, weather, $w$, and physical activity of the user, $a$, as shown by equation 1 .

$$
\text { usefulness }=e^{-f(d, w, a)}
$$

Ideally, the function $f$ should be determined based on actual measurements of the distance user travel in the various contexts (i.e. weather conditions in combination with transport modes). However in the current implementation, $f$ is simplified to the product of the distance, a factor determined by the weather, and a factor determined by the travel mode. Table 1 shows the values of these factors for illustration. Faster travel modes and better weather conditions are associated with smaller factors. Smaller factors in combination with the negative exponential curve induce that additional, further located items can also be considered as recommendations.

Also the availability of the user can be a limitation and is therefore checked by this model. Items that are not attainable within the time frame of the user's calendar (i.e., before the next appointment), given the user's transportation mode, are excluded as potential recommendation.
Table 1: The factors that influence the results of the distance punisher model, a factor determined by the travel mode and a factor for the current weather condition.

\begin{tabular}{|c|c|c|c|}
\hline standing/walking & running & cycling & car/train \\
\hline 10 & 5 & 3 & 1 \\
\hline \hline snow & rain & cloudy & sunny \\
\hline 6 & 4 & 2 & 1 \\
\hline
\end{tabular}

\subsubsection{Boredom punisher model}

Recommendations should not only reflect the personal preferences of the user (in a specific context), but also help the user to find surprisingly interesting items (s)he might not have otherwise discovered. E.g., recommending the user's favorite restaurant over and over again might not be useful. In the domain of recommender systems, serendipity is used as a measure of how useful and surprising the recommendations are (Herlocker et al., 2004). To increase the serendipity of the recommendations, the boredom punisher model favors the items that are new for the user at the expense of items that are already explored by the user (i.e. evaluated or selected for more information).

The information category of the item is an important characteristic that is taken into account by the model. The schedule of the movie theater for a movie that the user has already seen and evaluated is not useful, since people normally do not go to the movie theater twice to see the same movie. Likewise, recommendations for news articles that the user has already read are not desirable. In contrast, it might be interesting to provide information on a regular basis about the schedules and delays of a train that the user regularly catches. In conclusion, new, unexplored items receive the maximum score from the boredom punisher model. Items that the user has already interacted with, are disadvantaged by a specified penalty in accordance with the category of the item.

\subsubsection{Aggregating the item scores}

Each of the models discussed above generates a score that estimates the usefulness of each item based on the current context and preferences of the user. For each item, these scores are then aggregated into a single estimation of the usefulness, which is used to select a subset of the items within each information category as recommendations. Similar to the aggregation of the category scores, the item scores of the individual models are normalized and aggregated using a weighted average. In the current implementation, the weights are fixed and set to prioritize the model that estimates the usefulness based on the explicit prefer- 
ences of the user, i.e. the stated preferences model.

Also this aggregator can be extended with varying weights to anticipate the development and improvement of user profiles during service usage. For new users of the service who have a limited profile, the stated preferences and distance punisher model may be the most consistent models. As a user utilizes the recommendation service more often, his/her profile becomes more detailed, and as a result, the collaborative filtering, learned preferences, and boredom punisher model are able to make a valuable contribution. So the weights associated to the models can be made variable in accordance with the advancement of the user profile in order to generate more accurate recommendations.

So to conclude, the category score determines the importance of an information category and the corresponding amount of slots for that category in the recommendation list. For each information category, the items with the highest estimated usefulness are filling these slots and offered as recommendations to the users.

\section{EVALUATION}

To evaluate the usefulness and effectiveness of the application and the personal recommendations, a small user evaluation was performed. The test panel consisted of 16 test subjects ( 12 men and 4 women) who are representative for the target users of the applications. All test subjects were between 21 and 32 years old and make daily use of a smartphone. They were asked to download and install the application on their own smartphone and use it during one week to retrieve recommendations in their daily environment. To ensure that the test subjects are sufficiently familiar with the application for an evaluation after the test, we asked to use the application at least once a day and at least three times outdoors. The latter requirement stimulates test subjects to use the application on the move, or for exploring new places.

After one week, test subjects received a questionnaire to evaluate the application by means of 9 multiple choice questions and 3 open questions. The multiple choice questions consisted of statements that test subjects had to assess on a 5-point rating scale ranging from "1: totally disagree" to "5: totally agree". The goal of the open questions was to inquire for potential improvements or extensions to the application.

Figure 3 visualizes the answers to the most interesting multiple choice questions as histograms. The first histogram, Figure 3(a), indicates that all test subjects experienced the application as "easy to use". Be- cause of the automatic context recognition and the straightforward way to retrieve recommendations, no test subject provided a negative evaluation regarding the usability. Future work may comprise more comprehensive user tests to obtain a more detailed evaluation of the usability and functionality of the application and the interface by using adaptive user-interface techniques (Savidis and Stephanidis, 2010). The accuracy of the recommendations is assessed by asking the test subjects if the recommendations are interesting. Except for two people, the test subjects agreed with the statement that the recommendations of the application are really interesting for them, as illustrated in the second histogram, Figure 3(b). The test subject who totally disagreed with this statement had a data connection problem during the test, which explains why he did not receive (interesting) recommendations. The ability to help users discovering new and interesting information or POI, i.e. the serendipity of the recommendations, is assessed via the third histogram, Figure 3(c). Except for two people (one of them had a connection problem), test subjects confirmed that they can find new and interesting information or POI via the recommendations. The last histogram, Figure 3(d), gives an indication about how pleasant it is to use the application. Only three test subjects disagreed with the statement "I like to use the application".

To summarize, the application that offers contextaware recommendations based on the automaticallydetected context of the user, is easy to use. According to the test subjects, the personal recommendations are a valuable asset in the context of information retrieval: these recommendations are interesting and help them to discover new content and places.

Via the open questions, test subjects were asked if additional features should be added to the application, and which existing features should be removed. Four test subjects suggested to extend the friendfunctionality of the application. Besides adding and removing users from their friend list, they would like to see the context of their friends. They also mentioned the possibility to recommend items to friends and to see their friends' feedback on items. Three test subjects indicated that the items of the category "News" might be superfluous.

\section{CONCLUSIONS}

In this research, we investigated how the current context and activity of the user can be recognized based on sensor data and the accelerometer of his/her mobile device. The context-recognition framework first 


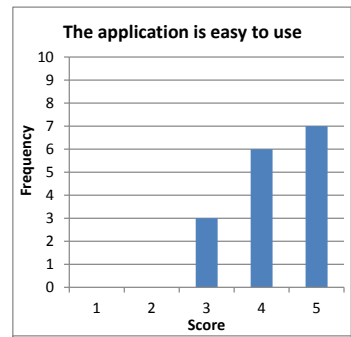

(a) Usability

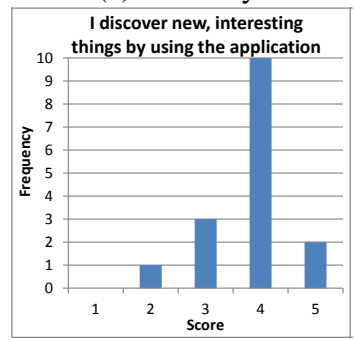

(c) Serendipity

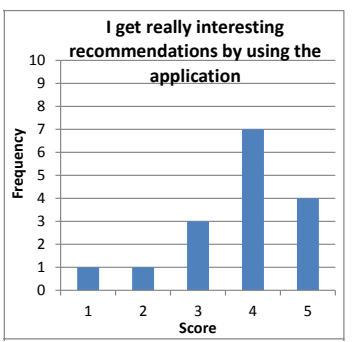

(b) Accuracy

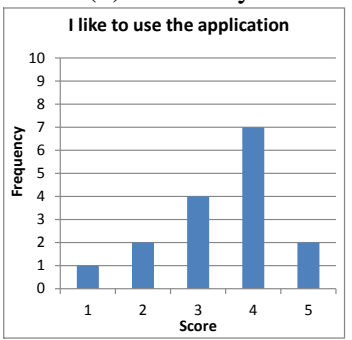

(d) Likeability
Figure 3: Histograms of the answers on the multiple choice questions of the user evaluation.

monitors and processes the sensor data to recognize basic activities or context changes. Then these successive basic activities are analyzed to recognize the overall context of the user. An evaluation of the framework proved that physical activities and the context of the user can be recognized with a high accuracy and that this contextual information can be valuable knowledge for a context-aware recommender system. Besides, the framework can be used for other applications, e.g., for monitoring the physical activities of the user in the context of health care.

A user study showed that context-aware recommendations are effective and helpful for discovering new places and interesting information. Moreover, user like to receive information tailored to their current needs and consider the recommender application as easy to use. These results confirm the necessity to adapt (mobile) applications and service to the activity and context of the user in order to improve the effectiveness and the user experience.

\section{REFERENCES}

Adomavicius, G., Sankaranarayanan, R., Sen, S., and Tuzhilin, A. (2005). Incorporating contextual information in recommender systems using a multidimensional approach. ACM Trans. Inf. Syst., 23(1):103145.

Bao, L. and Intille, S. (2004). Activity recognition from user-annotated acceleration data. In Ferscha, A. and Mattern, F., editors, Pervasive Computing, volume
3001 of Lecture Notes in Computer Science, pages 117. Springer Berlin / Heidelberg.

Biegel, G. and Cahill, V. (2004). A framework for developing mobile, context-aware applications. In Proceedings of the Second IEEE International Conference on Pervasive Computing and Communications (PerCom'04), PERCOM '04, pages 361-, Washington, DC, USA. IEEE Computer Society.

Breese, J. S., Heckerman, D., and Kadie, C. (1998). Empirical analysis of predictive algorithms for collaborative filtering. In Proceedings of the Fourteenth conference on Uncertainty in artificial intelligence, UAI'98, pages 43-52, San Francisco, CA, USA.

CultuurNet-Vlaanderen (2012). Uitdatabank developer tools. Available at http://tools.uitdatabank.be/docs.

Dodson, B. (2012). Wikilocation. Available at http://wikilocation.org/.

Foursquare (2012). Foursquare API. Available at https://developer.foursquare.com/.

Google (2012a). Geocoding API. Available at https://developers.google.com/maps/documentation/geocoding/.

Google (2012b). Places API. Available at https://developers.google.com/places/documentation/.

Herlocker, J. L., Konstan, J. A., Terveen, L. G., and Riedl, J. T. (2004). Evaluating collaborative filtering recommender systems. ACM Trans. Inf. Syst., 22(1):5-53.

HLN (2012). Rss news feed. Available at http://www.hln.be/rss.xml.

Iacono, M., Krizek, K., and El-Geneidy, A. (2008). Access to destinations: How close is close enough? estimating accurate distance decay functions for multiple modes and different purposes. Technical report, University of Minnesota, Twin Cities. Minnesota Department of Transportation. ref.: MN/RC 2008-11.

Kenteris, M., Gavalas, D., and Mpitziopoulos, A. (2010). A mobile tourism recommender system. In Proceedings of the The IEEE symposium on Computers and Communications, ISCC '10, pages 840-845, Washington, DC, USA. IEEE Computer Society.

Kwapisz, J. R., Weiss, G. M., and Moore, S. A. (2011). Activity recognition using cell phone accelerometers. SIGKDD Explor. Newsl., 12(2):74-82.

Lee, S.-W. and Mase, K. (2002). Activity and location recognition using wearable sensors. Pervasive Computing, IEEE, 1(3):24 -32.

Ravi, N., Dandekar, N., Mysore, P., and Littman, M. L. (2005). Activity recognition from accelerometer data. In Proceedings of the 17th conference on Innovative applications of artificial intelligence - Volume 3, IAAI'05, pages 1541-1546. AAAI Press.

Ricci, F. (2010). Mobile recommender systems. Information Technology \& Tourism, 12(3):205-231.

Savidis, A. and Stephanidis, C. (2010). Software refactoring process for adaptive user-interface composition. In Proceedings of the 2nd ACM SIGCHI symposium on Engineering interactive computing systems, EICS '10, pages 19-28, New York, NY, USA. ACM.

Tiete, Y., Schmitz, S., and Colpaert, P. (2012). iRail API. Available at http://project.irail.be/wiki/API/APIv1. 